\title{
Impact of analytic provenance in genome analysis
}

\author{
Shatavia S Morrison ${ }^{1}$, Roman Pyzh ${ }^{1}$, Myung S Jeon ${ }^{1}$, Carmen Amaro ${ }^{2}$, Francisco J Roig ${ }^{2}$, Craig Baker-Austin ${ }^{3}$, \\ James D Oliver ${ }^{4}$, Cynthia J Gibas ${ }^{1 *}$ \\ From 9th International Symposium on Bioinformatics Reseaerch and Applications (ISBRA'13) \\ Charlotte, NC, USA. 20-22 May 2013
}

\begin{abstract}
Background: Many computational methods are available for assembly and annotation of newly sequenced microbial genomes. However, when new genomes are reported in the literature, there is frequently very little critical analysis of choices made during the sequence assembly and gene annotation stages. These choices have a direct impact on the biologically relevant products of a genomic analysis - for instance identification of common and differentiating regions among genomes in a comparison, or identification of enriched gene functional categories in a specific strain. Here, we examine the outcomes of different assembly and analysis steps in typical workflows in a comparison among strains of Vibrio vulnificus.
\end{abstract}

Results: Using six recently sequenced strains of $V$. vulnificus, we demonstrate the "alternate realities" of comparative genomics, and how they depend on the choice of a robust assembly method and accurate ab initio annotation. We apply several popular assemblers for paired-end Illumina data, and three well-regarded $a b$ initio genefinders. We demonstrate significant differences in detected gene overlap among comparative genomics workflows that depend on these two steps. The divergence between workflows, even those using widely adopted methods, is obvious both at the single genome level and when a comparison is performed. In a typical example where multiple workflows are applied to the strain V. vulnificus CECT 4606, a workflow that uses the Velvet assembler and Glimmer gene finder identifies 3275 gene features, while a workflow that uses the Velvet assembler and the RAST annotation system identifies 5011 gene features. Only 3171 genes are identical between both workflows. When we examine 9 assembly/ annotation workflow scenarios as input to a three-way genome comparison, differentiating genes and even differentially represented functional categories change significantly from scenario to scenario.

Conclusions: Inconsistencies in genomic analysis can arise depending on the choices that are made during the assembly and annotation stages. These inconsistencies can have a significant impact on the interpretation of an individual genome's content. The impact is multiplied when comparison of content and function among multiple genomes is the goal. Tracking the analysis history of the data - its analytic provenance - is critical for reproducible analysis of genome data.

\section{Background}

Next generation sequencing has revolutionized the study of microbial genomics. To handle the millions of sequence read fragments produced by the next gen platforms, a variety of assembly approaches have been developed[1-3]. In most instances the assembler produces a set of contigs or scaffolds, which still leaves the genome

\footnotetext{
* Correspondence: cgibas@uncc.edu

'Department of Bioinformatics and Genomics, University of North Carolina at Charlotte, Charlotte, North Carolina 28223, USA

Full list of author information is available at the end of the article
}

in pieces. It is no longer common to completely finish and close a newly-sequenced genome. Usually, we evaluate the "success" of the assembly with two metrics: the number of contigs produced and the N50 value. Lower contig counts and higher N50 values are considered optimal. However, Parra et al. [4] and others [5] reported that choosing assemblies with higher N50 values frequently results in conserved genes going undetected in benchmark studies. If a gene is missed due to errors at the assembly stage it will not be annotated, leading to inconsistencies in downstream analyses.
() Biomed Central

(c) 2014 Morrison et al.; licensee BioMed Central Ltd. This is an Open Access article distributed under the terms of the Creative Commons Attribution License (http://creativecommons.org/licenses/by/4.0), which permits unrestricted use, distribution, and reproduction in any medium, provided the original work is properly cited. The Creative Commons Public Domain Dedication waiver (http://creativecommons.org/publicdomain/zero/1.0/) applies to the data made available in this article, unless otherwise stated. 
There have been several efforts to assess the quality of assemblies produced by de novo methods. GAGE [6] and the Assemblathon [7] projects provided goldstandard data sets and an environment for peer evaluation of assembly methods. Recently, next generation sequence assemblers were evaluated on bacterial datasets in the GAGE-B study. Magoc et al. [8] showed that a single library prep and deep (100x-250x) sequencing coverage is sufficient to capture the genomic content of most bacterial species, but demonstrated wide variation in the assemblies produced by different methods.

Analysis of genomes does not stop at assembly, however. There exist a wide range of methods for annotation of the assembled data. Genome annotation includes identification of the gene sequences within a contig, and assignment of function based on similarity to known genes or sequence patterns. Ab initio gene finders and methods for functional assignment each have their own associated uncertainty, and results from one method are unlikely to agree completely with those from another[5]. Assembly and annotation are the two major components of the bacterial genomics workflow, and there are an astonishing number of combinations of methods that can be used to carry out just these two steps.

When we survey the literature in microbial genomics, we find that investigators depositing microbial sequences have not come to a consensus on the best pipeline for genome analysis. Several different assemblers are in common use. Annotation methods may include anything from simply comparing the genome to a reference by using BLAST, to using $a b$ initio genefinders, to using integrated annotation pipelines provided by sequencing centers. Despite over a decade of literature on the performance of $a b$ initio genefinders and annotation pipelines [9-12] nearly any reasonable workflow seems able to pass peer review (Figure 1), and so the genome annotations found in the public databases vary widely in analytic provenance. Especially in the absence of reference genomes and bench work validation, the proliferation of analysis options can lead to inconsistencies (comparing apples to oranges) and ultimately to errors in biological interpretation. It is not possible to distinguish a true target, such as a gene that differentiates one genome from its near relatives, from an artifact introduced at the assembly or annotation steps. Yet investigators often seem to remain unaware of the impact of their choices, and how the selection of Glimmer[13] rather than GeneMark [14] (for example) may result in a greatly altered story when they begin to analyze the apparent content of a newly sequenced genome. Figure 1 is a summary of the major elements of current genomic workflows based on a census of 2013 bacterial genome announcements in recent issues of the journal GenomeA (American Society of Microbiology) [15].

In recent years, the biomedical research community has increasingly recognized the failure of many studies to achieve reproducibility [16] in data analysis protocols. In experiments using NGS data, which rely entirely

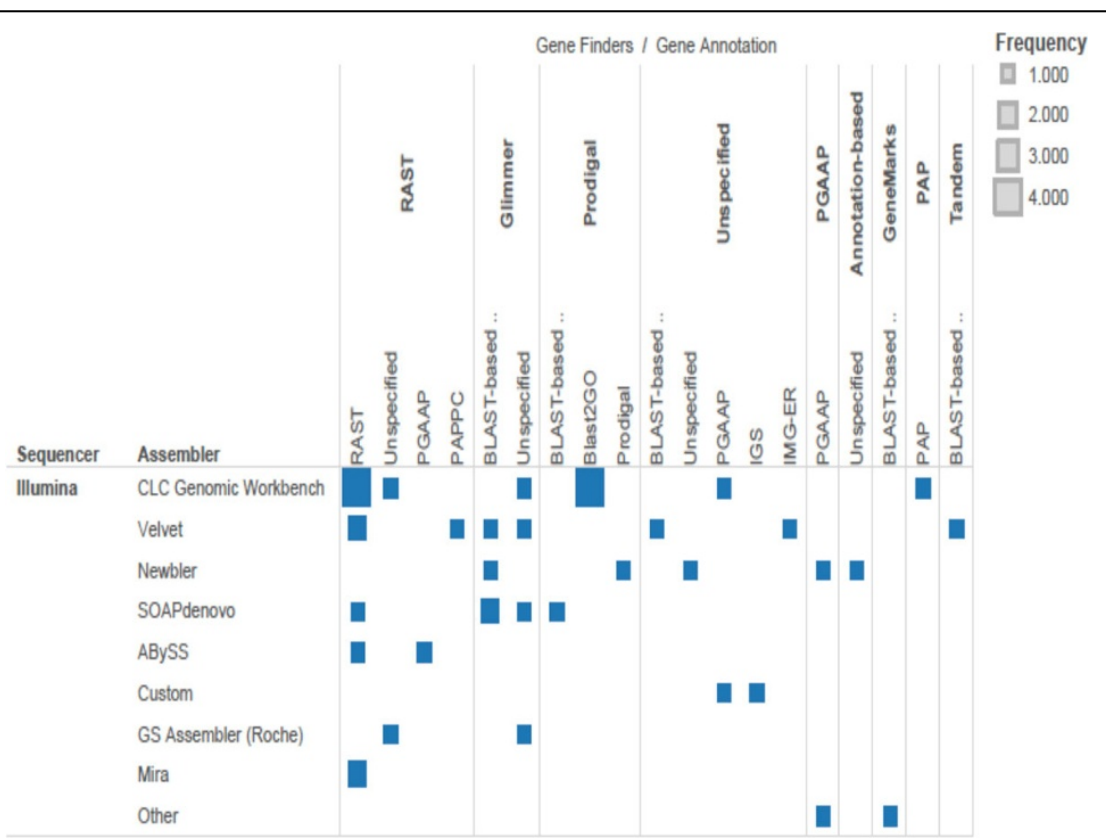

Figure 1 Crosstab map of frequency levels of assembler and annotation method applied to Illumina data. Figure shows the frequency of the number of times a particular combination of assembler and annotation method was used in 40 Genome Announcements from the September 2012 Vol. 194, Issues 17 and 18 of Journal of Bacteriology and January and February 2013 Vol. 1., Issue 1 of Genome Annoucements. 
upon computational analyses for interpretability, the ability to trace the history of and reproduce data analysis is especially critical $[17,18]$. Innovation in this domain is rapid and is ongoing, and best practices for reproducibility in bioinformatics are increasingly widely discussed[19]. The concept of workflow, originally used to describe business processes, began to be used more broadly in bioinformatics with the advent of high throughput sequencing in the early 2000s [20]. Systems such as Galaxy [21], Taverna [22], and MOLGENIS [23], among others, have made reproducible workflows more accessible to users of bioinformatics software, and many workflow systems now include the means for tracking analytic provenance,[19]but it is clear from our survey of the literature that these innovations have yet to completely penetrate to end users of assembly and annotation methods for microbial genome sequencing.

In this study, we assess the scope of the data interpretation problem caused by variation in pipeline choices. Starting with five $V$. vulnificus strains for which paired-end Illumina sequence was collected, and one $V$. vulnificus genome with a high quality finished sequence that has been continually revised and updated [24], we apply wellregarded assembly and annotation methods, in different combinations, to the data. We have chosen to focus on only a few of those most popular methods in each category, because workflow construction from multiple options is a combinatorial problem. The case study data demonstrate the influence of choices made during the assembly and annotation stages on biological interpretation of newly sequenced genomes. Vibrio vulnificus is a bacterium commonly found in estuarine waters and mollusks. It is responsible for $95 \%$ of all deaths resulting from seafood consumption in the United States [25]. There are both clinical isolates and environmental genotypes associated with this bacterium, making it a prime candidate for comparative genomics study. In the present study, we demonstrate the direct impact of parameter and method choices on the biologically relevant products of a comparative genomics analysis among strains of Vibrio vulnificus. Comparative analysis of gene content and function is a highly relevant case study, as this analysis is a popular protocol among microbiologists, and has been shown to be more effective than MLST for bacterial strain characterization [26]. The results highlight the influence of the assembly and annotation pipeline on comparative content and function analysis, and emphasize the need for contributors of genomic data to provide complete information about the analytic provenance of their assembled and annotated genomes, and for consistent workflows, justified by benchmark testing where possible, to be used throughout a project. Workflows used in this analysis were constructed in the Taverna workflow system, and are available as a workflow pack at http://MyExperiment. org. [http://www.myexperiment.org/packs/625.html].

\section{Results}

Workflow dependent outcomes in a simulated assembly case

As a basis for choosing an appropriate analysis pipeline for newly sequenced $V$. vulnificus genomes, we first generated simulated read data from the genome of $V$. vulnificus CMCP6. This genome was initially sequenced using Sanger sequencing and a traditional genome finishing approach in 2003, [27] and was partially sequenced and completely reannotated in 2011[24]. While the original annotation relied primarily on a combination of $a b$ initio genefinders, the subsequent reannotation used additional information from closely homologous genomes and public databases of curated gene sequence patterns. The published sequence and annotations for $V$. vulnificus CMCP6 are still not exhaustively validated by transcriptome data, but they are the most heavily curated of the available Vibrio vulnificus genome annotations, and therefore we use them as the frame of reference for evaluating different approaches to assembly and annotation.

We performed de novo sequence assemblies of the simulated data with Velvet (V), ABySS (A), and SoapDenovo (S). GeneMark.hmm (GeneMark)[14] and RAST[28] were then used to identify gene sequences for each contig set. We used OrthoMCL[29] with a stringent similarity cutoff to cluster predicted genes with their counterparts in the 2011 V. vulnificus CMCP6 annotation.

The contig counts observed were 205, 144, and 269 for the V, A, and S assemblies, respectively. Table 1 summarizes gene counts obtained for each assembly followed by each gene annotation method, for the simulated V. vulnificus CMCP6 genomes. To avoid ambiguity, the percentage of genes recovered refers only to predicted genes, which clustered uniquely with one gene in the reference annotation. Less than $1 \%$ of predicted genes cluster with apparent paralogs in the reference genome when clustered at a $95 \%$ threshold. The results presented in Table 1 suggest that, while the Velvet assembler [1] does not assemble the simulated data into the smallest number of contigs, it produces the most accurate

Table 1 Assembly and Annotation of V. vulnificus CMCP6.

\begin{tabular}{llll}
\hline Assembly method & Velvet & ABySS & Soap \\
\hline \# of contigs & 205 & 144 & 269 \\
\hline Assembly+RAST performance & & & \\
\hline \# of genes predicted & 4684 & 5095 & 4720 \\
\hline \# of genes with match in CMCP6 & 3890 & 3777 & 3863 \\
\hline$\%$ of known genes recovered & $91.8 \%$ & $89.2 \%$ & $91.2 \%$ \\
\hline Assembly+Genemark performance & & & \\
\hline \# of genes predicted & 4761 & 5051 & 4833 \\
\hline \# of genes with match in CMCP6 & 4019 & 3754 & 3844 \\
\hline$\%$ of known genes recovered & $94.9 \%$ & $88.6 \%$ & $90.7 \%$ \\
\hline
\end{tabular}


assembly of the simulated V. vulnificus CMCP6 data. Velvet, in combination with the GeneMark[14]ab initio genefinder, may produce the best results on novel $V$. vulnificus sequence data. This type of simple twostep workflow is representative of genome analysis workflows found in the genome announcements surveyed in Figure 1. However, it should be noted that the best-performing workflow still resulted in a loss of over 200 previously annotated genes, when reanalyzing simulated V. vulnificus CMCP6 data.

\section{Workflow dependent outcomes on novel genome data}

The published Vibrio vulnificus genomes are mainly composed of 2 circular chromosomes, and some are known to have plasmids. The size of the $V$. vulnificus genome is estimated at 5.6 Mb-5.8 $\mathrm{Mb}$ of DNA, and this size is consistent among known strains. The newly sequenced isolates $V$. vulnificus CIP8190, CECT5198, CECT4606, CECT5763, and CECT4886 are all known to have 2 chromosomes and 2,3,1,2, and 2 plasmids, respectively. Table 2 describes each genome used in this study and its genomic characteristics, as well as the number of sequence reads available for each genome.

Our analysis here is primarily focused on the performance of the assembly and annotation steps typically used during the construction of a draft genome. Biological findings for these genomes will be the focus of another manuscript, currently in preparation. Using the workflow framework shown in Figure 2, we assembled contig sets and annotation sets for each $V$. vulnificus strain. After the removal of sequence reads containing ' $N$ ' characters, and random sampling of read pairs to obtain 100x genome coverage based on the Lander Waterman statistic [30], there were 11,400,000 paired end reads in the final read sets for each of the newly sequenced strains. The same coverage depth was simulated for $V$. vulnificus CMCP6.

Using the same de novo assemblers we applied to the simulated data set, we constructed contig sets ranging in size from 180-630 contigs for each of the input genomes. Table 3 summarizes the output of Velvet, Soap,

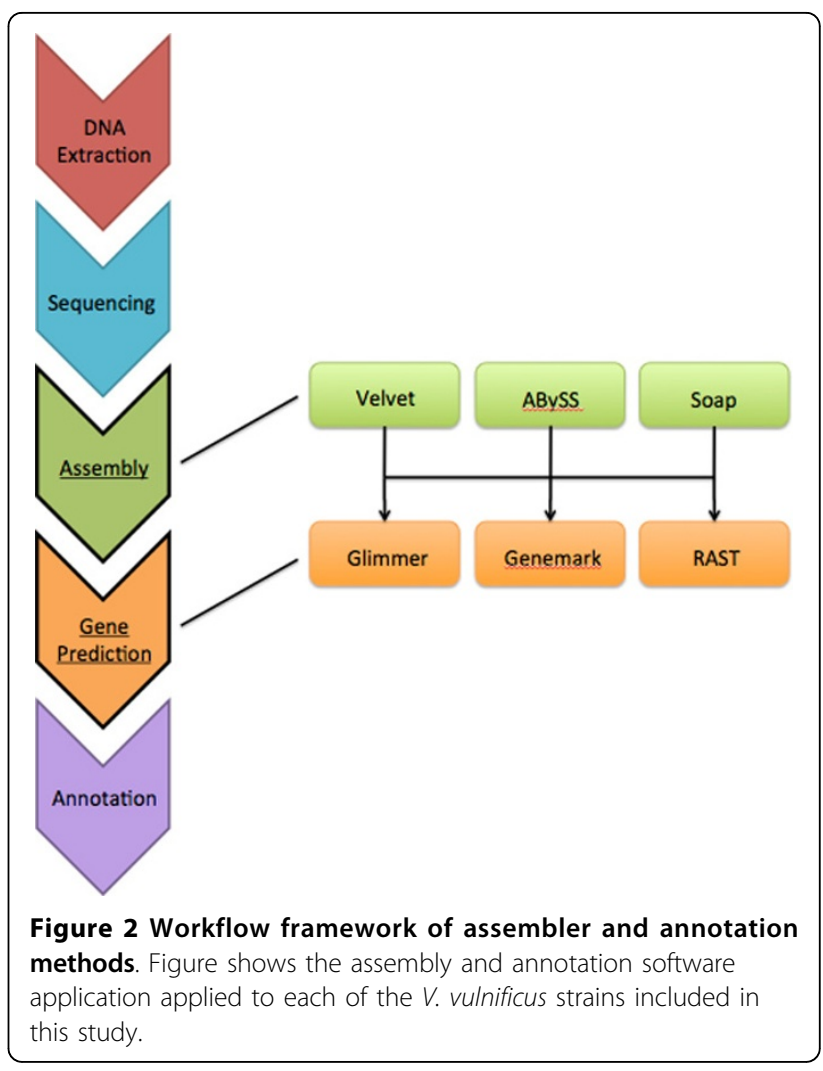

and ABySS assemblies for each $V$. vulnificus strain. We then used MuMMer 2.3[31] to align the contig sets for each strain, using an all-against-all alignment to identify contigs that were similarly constructed between the assemblers. Contig pairs that exceeded coverage and sequence identity cut-offs of $95 \%$ were identified as similarly constructed. Figure 3 summarizes the conservation of contigs across assemblies. Although counts varied from genome to genome, we observed on average 43 contigs constructed by all three assemblers, 133 found by any combination of two of the three assemblers, and 445 contigs that were uniquely constructed by a specific assembler.

Table 2 Genomic Characteristics of Vibrio vulnificus CMCP6, CIP8190, CECT5198, CECT4606, CECT5763, and CECT4866.

\begin{tabular}{|c|c|c|c|c|c|c|}
\hline Genomic Characteristic & CMCP6 & CIP8190 & CECT5198 & CECT4606 & CECT5763 & CECT4866 \\
\hline Biotype & 1 & 2 & 2 & 2 & 2 & 2 \\
\hline Genotype & C & $C$ & $E$ & $E$ & $E$ & $C$ \\
\hline Chr Number & 2 & 2 & 2 & 2 & 2 & 2 \\
\hline Plasmid Number & None & 2 & 3 & 2 & 2 & 2 \\
\hline Average $\mathrm{G}+\mathrm{C}$ content & $46.6 \%$ & $46.5 \%$ & $46.5 \%$ & $46.2 \%$ & $46.3 \%$ & $46.5 \%$ \\
\hline \# of reads generated & $6620286^{*}$ & 26869740 & 14366914 & 23523786 & 18852452 & 33792718 \\
\hline N50 for Velvet & 196375 & 71778 & 60906 & 316446 & 51991 & 65142 \\
\hline N50 for ABySS & 187671 & 57867 & 66098 & 154882 & 54273 & 64876 \\
\hline N50 for Soap & 196396 & 71391 & 62139 & 165040 & 52087 & 65144 \\
\hline
\end{tabular}


Table 3 Total number of contigs assembled for V. vulnificus CMCP6, CIP8190, CECT5198, CECT4606, CECT5763, and CECT4866.

\begin{tabular}{lccc}
\hline Strain & Velvet & Abyss & Soap \\
\hline V. vulnificus CMCP6 & 205 & 144 & 269 \\
\hline V. vulnificus CIP8190 & 284 & 364 & 507 \\
\hline V. vulnificus CECT5198 & 302 & 289 & 448 \\
\hline V. vulnificus CECT4606 & 129 & 148 & 267 \\
\hline V. vulnificus CECT5763 & 492 & 743 & 845 \\
\hline V. vulnificus CECT4866 & 404 & 366 & 519 \\
\hline
\end{tabular}

In our analysis of the novel Vibrio vulnificus genomes, we included the Glimmer3.0[13] ab initio gene-finding method in addition to GeneMark and RAST. Glimmer3.0 is demonstrated to be approximately $96 \%$ accurate in gene identification,[13]which is similar to the accuracy that we observed for GeneMark in the CMCP6 case study above. In Table 4, we summarize the gene predictions by each of the three prediction methods for each of the three assemblies constructed for each $V$. vulnificus strain. We find that RAST and GeneMark tend to identify more regions as putative genes sequences than Glimmer for these strains. However, this is not a case of simple over-prediction, since the Glimmer gene sequences are not strictly a subset of the predictions by other methods. As an example, in Figure 4 we detail the number of gene overlaps between all possible assembly-to-annotation permutations for $V$. vulnificus CECT4606.

Figure 4 summarizes the gene overlaps for Vibrio vulnificus CECT4606 datasets for different genefinders applied to assemblies. Gene overlaps are defined as two genes identified by different pipelines, which have the

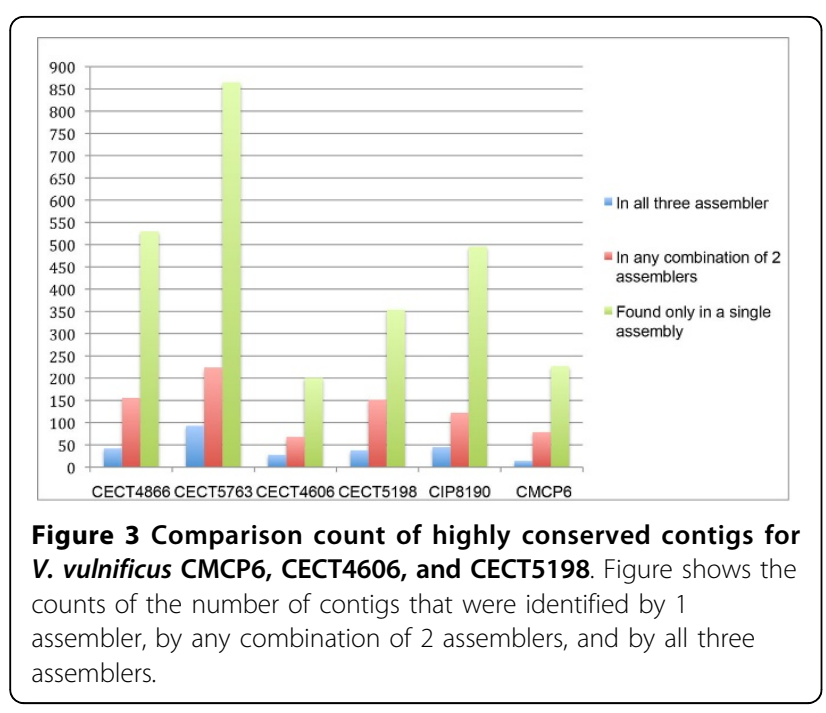

Table 4 Total number of genes predicted for $V$. vulnificus strains included in this study.

\begin{tabular}{lccc}
\hline A.) & & & \\
\hline Glimmer & Abyss & Soap & Velvet \\
\hline V. vulnificus CMCP6 & 3226 & 3042 & 3047 \\
\hline V. vulnificus CIP8190 & 3233 & 3030 & 3032 \\
\hline V. vulnificus CECT5198 & 3289 & 2973 & 2977 \\
\hline V. vulnificus CECT4606 & 3465 & 3275 & 3275 \\
\hline V. vulnificus CECT5763 & 3253 & 3079 & 3083 \\
\hline V. vulnificus CECT4866 & 3301 & 3024 & 3031 \\
\hline B.) & & & \\
\hline Rast & Abyss & Soap & Velvet \\
\hline V. vulnificus CMCP6 & 5095 & 4720 & 4684 \\
\hline V. vulnificus CIP8190 & 4963 & 4600 & 4623 \\
\hline V. vulnificus CECT5198 & 5021 & 4554 & 4563 \\
\hline V. vulnificus CECT4606 & 5315 & 5015 & 5011 \\
\hline V. vulnificus CECT5763 & 5038 & 4732 & 4752 \\
\hline V. vulnificus CECT4866 & 5035 & 4605 & 4631 \\
\hline C.) & & & \\
\hline Genemark & Abyss & Soap & Velvet \\
\hline V. vulnificus CMCP6 & 5051 & 4833 & 4761 \\
\hline V. vulnificus CIP8190 & 5084 & 4912 & 4787 \\
\hline V. vulnificus CECT5198 & 5187 & 4795 & 4710 \\
\hline V. vulnificus CECT4606 & 5500 & 5311 & 5189 \\
\hline V. vulnificus CECT5763 & 5489 & 5346 & 5062 \\
\hline V. vulnificus CECT4866 & 5243 & 4931 & 4839 \\
\hline
\end{tabular}

same stop signals and strand orientation on the same contig sequence. In prokaryotes, $a b$ initio genefinder predictions are known to be least reliable for very short genes[32]. As an example, in Figure 5, we show the distribution of gene lengths for consensus and non-consensus genes in a case were the RAST and Glimmer genefinders were both applied to the genome of $V$. vulnificus CECT4606, with the SoapDenovo assembler. Genes of length 500 and below are nearly entirely nonconsensus genes, while genes above 700 in length are nearly entirely in consensus between the two methods. It is in the region between 500 and 700 nucleotides where potentially ambiguous cases are found, involving several hundred genes. Glimmer tends to predict fewer genes that are outside the common "core" of predictions produced by all three genefinders. It is possible that this reflects greater accuracy, or it may be that Glimmer alone is more conservative in its gene-identification model. RAST (which uses Glimmer in an initial annotation pass) and GeneMark both make, and agree upon, predictions that are excluded from the Glimmer prediction set. It is possible that these two methods are potentially capturing more species-specific genes. 


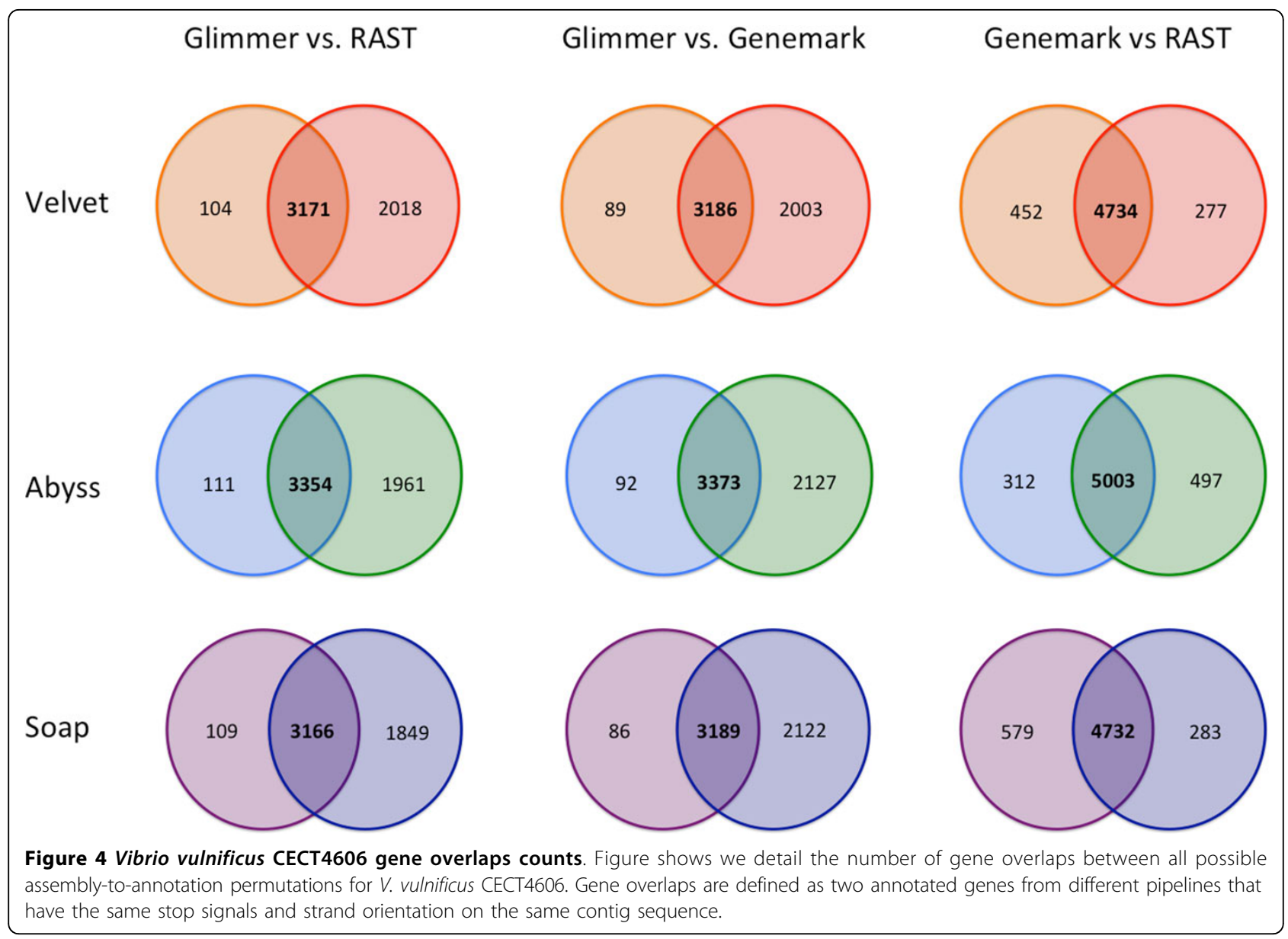

\section{Workflow dependent outcomes in functional analysis}

An archetypal result presented in genomic analyses is the categorization of genes into functional categories. This type of analysis is frequently used to draw conclusions about the energy sources an organism can use for survival, or about the genome's capacity to code for systems related to pathogenicity. To illustrate the impact of workflow choice on interpretation of functional content, we performed a comparative analysis among the results of six assembly-to-annotation workflows applied to the genome of $V$. vulnificus CECT4866, refer to Table 5. We used the GenoSets[33] analysis system to perform the comparison of analysis outcomes, treating the annotation set produced by each workflow as if it were an independent "genome".

Each workflow's gene set was assigned Gene Ontology (GO) terms [34,35] as described in Cain et al., 2012[33]. $\mathrm{GO}$ categories and individual genes having functionality significant enrichment or depletion between the various annotation versions were identified using the Gene Ontologizer[36]. See additional file 1 which summarizes the complete GO enrichment set for each of the workflow combinations examined. We first compared annotations produced by a workflow that used the Velvet assembler, followed by either Glimmer or GeneMark. 134 genes appeared in the Glimmer predictions, but not in the GeneMark predictions, resulting in the appearance of statistically significant enrichment or depletion in two GO functional categories. Deoxyribose phosphate metabolic process and deoxyribose phosphate catabolic process $\mathrm{p}$ values were 0.0066 and 0.0072 , respectively. 120 genes were identified solely with GeneMark annotations. Use of GeneMark resulted in the appearance of enrichment in GO terms associated with response to stress and iron ion binding, with $\mathrm{p}$-values at $5.99^{\mathrm{E}-12}$ and 0.0017 , respectively. The GO terms associated with iron utilization are especially of interest in the context of Vibrio vulnificus genomics, because as a pathogen it is especially dangerous to hosts in a condition of iron overload[37]. Ironprotein binding and stress response are potentially regarded as factors contributing to $V$. vulnificus's pathogenicity. Several studies have reported on the correlation between $V$. vulnificus infections and increased levels of iron in animal models and infected individuals[25,37,38]. Wright et al.[37] showed the injecting mice with iron prior to $V$. vulnificus infection significantly lowered the 


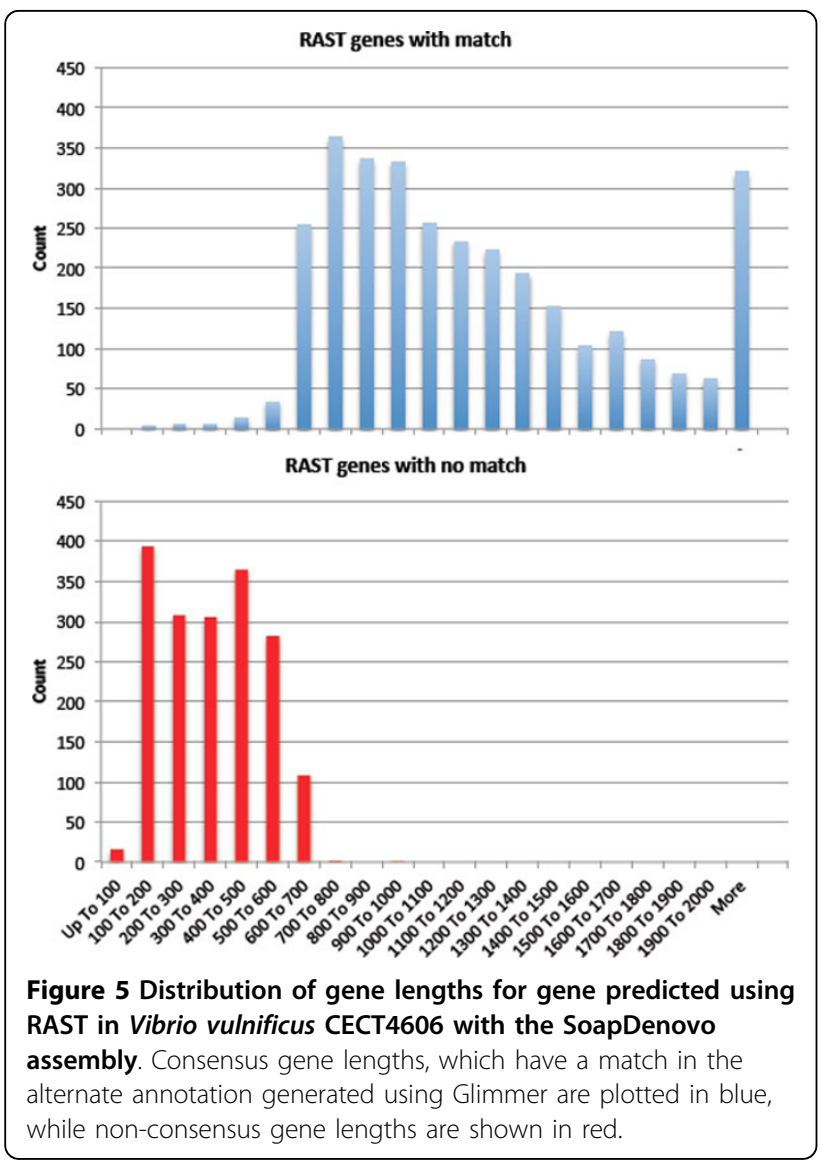

$\mathrm{LD}_{50}$. Amaro et al.[38] showed that after the injection of $V$. vulnificus to an iron-overload mice, they always died within a 48 hour period of inoculation. In this case, changing the assembly-to-annotation analysis pipeline results in a significant change in detected gene content, in a category that is directly relevant to the biology of the pathogen.

We next examined pipelines using the ABySS assembler followed by RAST or Glimmer. 1880 genes were unique to the RAST annotation. Of these, 132 significant GO enrichment terms were identified. In this set we find both iron-binding protein and terms associated with response to stress, again suggesting that the choice of assembly-to-annotation pipeline has the potential to significantly alter biological interpretation. Only 148 gene clusters were unique to the Glimmer set, and only 5 functional categories showed apparent statistically significant enrichment. Comparison of RAST and GeneMark annotations on a SOAPdenovo assembly resulted in approximately 10 statistically significant differences in functional content in either direction, although none of these categories were identified as significant to the biology of $V$. vulnificus in a previous study[39].

While these results are not conclusive, they indicate that at least in the case of $V$. vulnificus, RAST or GeneMark predictions may best reflect the presence of genes in key functional categories, known to be significant in the biology of these organisms.

\section{Workflow dependent outcomes in genome content comparison}

Another archetypal figure found in nearly every comparative genomics analysis paper is the Venn diagram or its conceptual equivalent. The Venn diagram provides a convenient method to summarize what the microbiologist really wants to know: what is in strain (or species) A that makes it function differently from strain B? In Figure 6 , we show the effect on this commonly-generated analysis product when different assembly-to-annotation pipelines are used to generate the input data. As an illustrative example, we performed gene content comparisons between $V$. vulnificus strain CMCP6 (clinical genotype) and strain CECT5198 (environmental genotype). In each comparison, the same assembly-to-annotation pipeline was used on each of the genomes being compared. We tested four combinations of assembler and genefinder. In Figure 6, we show that the majority of differences are seen when different annotation methods are used. In contrast, when different assemblers are used with the same annotation method, the number of differential genes are highly conserved. Given the large number of non-identical genes found when different pipelines are used on the same genome, as we saw in the previous examples, the result is as expected - the valuable biological "end product", the set of differentiating genes around which the biologist will build their scientific conclusions, can vary by dozens if not hundreds of members.

Table 5 Workflow descriptions used in differential functional analysis of Vibrio vulnificus CECT4866.

\begin{tabular}{|c|c|c|c|}
\hline Workflow Assignment & Assembly Type & Annotation Method & Number of genes \\
\hline A & Velvet & Glimmer & 3031 \\
\hline B & Velvet & Genemark & 4839 \\
\hline C & Abyss & Rast & 5035 \\
\hline D & Abyss & Glimmer & 3301 \\
\hline E & Soap & Genemark & 4931 \\
\hline $\mathrm{F}$ & Soap & Rast & 4605 \\
\hline
\end{tabular}




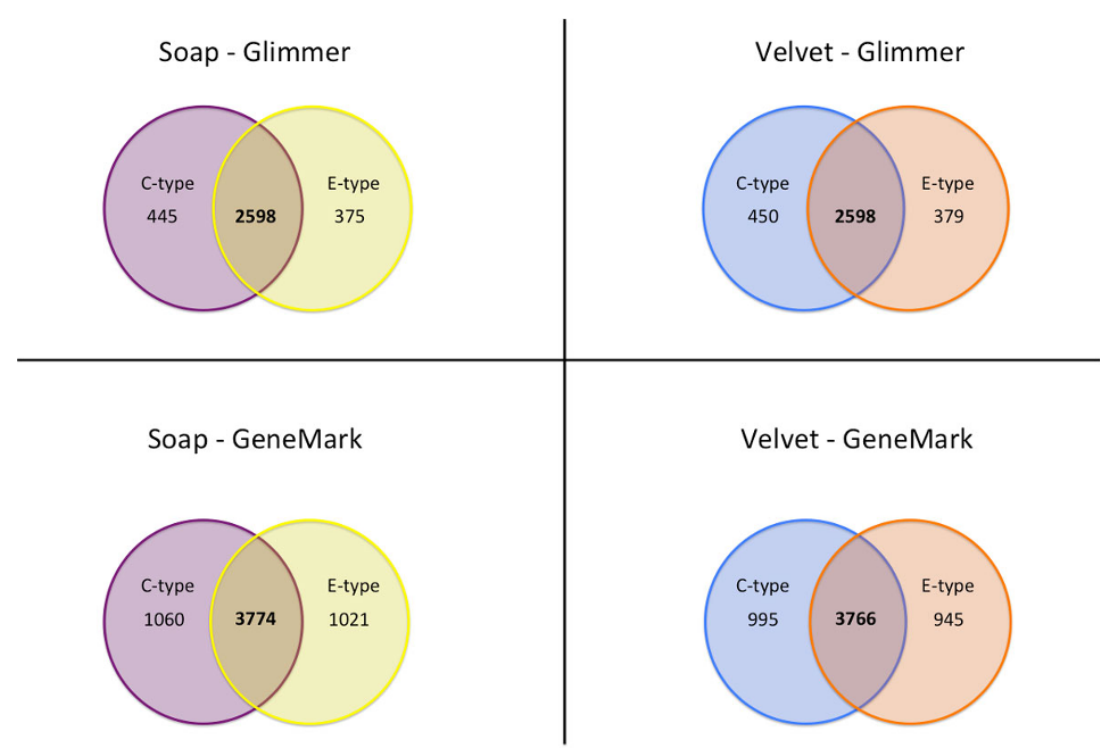

Figure 6 Genome content comparison for Vibrio vulnificus CMCP6 and CECT5198. Venn diagrams represent the differential and shared gene counts between V. vulnificus CMCP6 and V. vulnificus CECT5198 using the Velvet and SoapDenovo assemblies, each with Glimmer and GeneMark annotations.

\section{Discussion}

Many factors can have an impact on the assembly of next generation sequence data. Typical information captured about the provenance of sequence data focuses on laboratory procedures and conditions, as we see in the MIGS [40] standard for genomic data, or in the experiment information preserved in, for example, the NCBI's Gene Expression Omnibus[41]. However, assuming that samples were properly handled and prepared in the laboratory, and that procedures and conditions are consistent, there is still an entire layer of provenance information to be considered. Here, we have considered the analytic provenance of genome sequence data, that is, the computational steps that are executed to process the data and to attach features and functional information that allows for interpretation.

Despite an attitude on the part of researchers and publishers that microbial genome analysis is a solved problem, application of multiple assembly-to-annotation pipelines to the same data demonstrates that analysis outcomes are heavily dependent on pipeline choice. These choices carry forward into comparative content analysis and functional analysis of genomes, and have the potential to significantly impact scientific conclusions.

It is now typical to report on novel microbial genomes in terse genome announcements, abstract-style papers that give little information about parameterization and execution of bioinformatics processes. A survey of these typical papers shows that a wide variety of genome analysis pipelines using combinations of bioinformatics tools, from simple to sophisticated, will pass peer review. However, on closer examination typical pipelines do not produce identical or even similar results. And while in the hands of trained bioinformaticians, the pipelines we tested in this paper may be fine-tuned to produce somewhat more accurate results, the literature surveyed suggests that this is not what is happening "on the ground" in analysis of bacterial genomes. If the protocols outlined in recent genome reports are accurate, in many cases these protocols are no more complex than the simple one assembler, one genefinder workflows we have analyzed here.

\section{Conclusions}

While in many cases, there is not a standardized set of assembly and gene annotation tools as well as pipeline workflows for novel genome assemblies and annotations available, we recommend that creators of microbial genome datasets consider the following strategies to ensure high quality, reproducible analysis. First, if possible, benchmark proposed analysis pipelines using simulated data derived from a high-quality genome sequence that is as closely related to the novel sequences as possible[42]. Second, maintain an awareness of the variability of assemblyto-annotation results. Perform parallel analyses and assess downstream results for pipeline dependence. Finally, maintain a detailed record of the analytic provenance of the secondary data generated from your raw sequence reads, including pipeline steps and parameters.

\section{Methods}

\section{Genome sequencing}

$V$. vulnificus strains were sequenced at The Genome Analysis Centre (TGAC) using the Illumina HiSeq2000 
platform. Sequencing was carried out on pooled libraries, using pools of 12 strains in one lane of the Illumina HiSeq 2000, and producing on average 100 base pair paired-end reads.

\section{Sequencing simulation}

V. vulnificus CMCP6 chromosome 1 and 2 genome sequences were used to construct a simulated data set of 100 base pair paired-end reads. The simulated read set was constructed with ART version 1.5.0 using the program art_illumina[43]. The simulation parameters used were as follows: data type "paired end", read length "100", fold coverage "100", and quality score " 20 " (forward and reverse sequence reads). This dataset was used as a benchmark to evaluate the performance of the de novo assemblers, gene prediction algorithms, and annotation methods to reproduce the published sequence and annotations of the CMCP6 genome. $V$. vulnificus CMCP6 was recently re-annotated and is regarded as the most complete and accurate of the published $V$. vulnificus genomes at the time of this writing.

\section{Data cleansing}

FastQC was used to evaluate the quality of the sequence reads for each strain[44]. Any repetitive sequence identified by FastQC was removed from the dataset using an in-house perl script. Reads containing ' $N$ ' characters were also removed. After the data-cleansing steps were completed we sampled a subset of reads for each strain that was equivalent to $100 x$ coverage based on the Lander and Waterman statistic[30]. After the datacleansing steps were completed each newly sequenced isolate read set contained 11,400,000 paired reads. In the case of $V$. vulnificus CMCP6, the ART sequencing simulation program art-illumina generated 6,620,286 paired reads for CMCP6 using an identical threshold. This difference may be due to use of an alternative mathematical formula for calculating genome coverage in ART.

\section{Sequence assembly}

Initially, each read set was assembled with VelvetOptimiser version 2.2.0 and Velvet 1.0.17 in order to identify an optimal kmer value for assembly and construct an initial contig set. The optimal kmer values were 79 for V. vulnificus CIP8190 and CECT5763, 83 for V. vulnificus CMCP6 and 87 for $V$. vulnificus CECT5198, CECT4606, and CECT4886. The VelvetOptimiser parameters were then used to initiate the Velvet assembler. The VelvetOptimiser hash value (kmer) was set to a range of 73 to 93 . The read description parameter was set to "-shortPaired". The VelvetOptimiser optimal kmer value was also used as the input kmer value for ABySS version 1.2.6 (abyss-pe) and SOAPdenovo version
SOAPdenovo127mer. The default paired-end parameters were used for both assemblers.

\section{Contig comparison}

MuMMER 2.3[31] was used to create sequence alignments between assembled contigs, within collections of assemblies for the same genome and among genomes.

\section{Genome annotation}

$A b$ initio gene-finding and functional annotation for each contig set was performed using the in-house workflow MAP (manuscript in preparation) constructed in the Taverna workflow management system[22]. This workflow executes parallel assembly-to-analysis pipelines on a genomic data set. The $a b$ initio annotation methods implemented include Glimmer3.02, GeneMark.hmm and the Rapid Annotation using Subsystem Technology (RAST)[28] web service. The training model used for $a b$ initio gene-finding with Glimmer and GeneMark was constructed based on published Vibrio vulnificus annotations available in the NCBI database. The RAST web service parameters used were as follows: the genetic code was set to 11 for bacteria, taxonomy id was set to 672 for genus Vibrio, and the corresponding sequencing statistics for each strain were provided to the web service.

\section{Ortholog identification}

OrthoMCL[29]was used to cluster gene predictions with reference genes in the Vibrio vulnificus CMCP6 genome. For this application a cluster threshold of $95 \%$ identity was used. OrthoMCL[29]was also used to make connections between orthologs among sequenced Vibrio vulnificus strains, with a clustering threshold of $70 \%$ identity.

\section{Functional annotation}

Gene ontology (GO) terms were assigned using the BLAST2GO software[45]. BLAST2GO was used to perform a BLASTP against the $\mathrm{nr}$ (non-redundant) protein database, with e-value cut-off set to $1^{\mathrm{E}-6}$. GO annotations were assigned based on the BLAST2GO database version b2g_mar13. BLAST2GO assigns GO terms based on a weighted system of evidence codes.

\section{Content and functional comparison}

For comparison of assembly-to-annotation workflow outcomes and for comparisons of genomic content, we used the GenoSets software application[33]. The annotations produced by each workflow were loaded into the GenoSets application, which enables comparisons among multiple genomes. Each alternate annotation was treated as a separate "genome" in the GenoSets system. We followed the same gene clustering procedure used in Morrison et al. 2012[39] to define sets of genes that differentiate between genomes. To differentiate between 
the assembly-to-analysis pipeline outcomes, the approach was modified to reflect the expectations that gene sequences arising from different analysis workflows would be highly similar. OrthoMCL clustering was performed against the Vibrio vulnificus reference genome CMCP6 and clusters were formed based on a shared sequence similarity of $90 \%$, instead of the OrthoMCL default parameter of $50 \%$. The increase in stringency to $90 \%$ shared sequence similarity results in tightly constrained gene clusters, and allows for the possible of identified genes on the ends of contig that may have not been predicted in their entirety.

\section{Additional material}

Additional file 1: Excel document that summarizes the complete list of GO enrichment terms for the workflow description listed in

Table 5. GO enrichment terms were defined as significant with a p-value above .005 cut-off.

\section{Competing interests}

The authors declare that they have no competing interests.

\section{Authors' contributions}

SSM and CJG conceived the study. SSM designed and developed the approaches for the study. SSM and MSJ perform the bioinformatics analysis. RP performed the survey of microbial genome announcements and designed model to keep track of assembly and annotation methods used in survey. SSM, RP, MSJ, CA, FJR, CBA, JDO, and CJG gave final approval of the version to be published. CA and JDO supplied Vibrio vulnificus isolates for sequencing. CBA provided Vibrio vulnificus sequencing data sets used in this study.

\section{Acknowledgements}

We would like to thank Christina J. Castro for assisting in the preparation of this manuscript.

\section{Declaration}

Funding for this project was provided by NSF SI2:SSE award number 1047896 to CJG and by a DOE GAANN Fellowship for SSM. Programa Consolider-Ingenio 2010 CSD 2009-00006 from MICINN (Spain) provided funding for CA and FJR. The funding agencies had no role in the design, collection, analysis or interpretation of the data, in preparation of the manuscript, or in the decision to submit the manuscript for publication. JDO was supported by the Cooperative State Research, Education, and Extension Service, U.S. Department of Agriculture, Award No. 2009-03571 and any opinion, findings, conclusions, or recommendations expressed in this publication are those of the authors and do not necessarily reflect the views of the U.S. Department of Agriculture.

This article has been published as part of BMC Genomics Volume 15 Supplement 8, 2014: Selected articles from the 9th International Symposium on Bioinformatics Research and Applications (ISBRA'13): Genomics. The full contents of the supplement are available online at http://www biomedcentral.com/bmcgenomics/supplements/15/S8.

\footnotetext{
Authors' details

'Department of Bioinformatics and Genomics, University of North Carolina at Charlotte, Charlotte, North Carolina 28223, USA. ${ }^{2}$ Department of Microbiology and Ecology, University of Valencia, Valencia, Spain. ${ }^{3}$ Centre for Environment, Fisheries, and Aquaculture Science, Weymouth, Dorset, UK. ${ }^{4}$ Department of Biology, University of North Carolina at Charlotte, Charlotte, North Carolina 28223, USA.
}

Published: 13 November 2014
References

1. Zerbino DR, Birney E: Velvet: algorithms for de novo short read assembly using de Bruijn graphs. Genome Res 2008, 18(5):821-829.

2. Luo R, Liu B, Xie Y, Li Z, Huang W, Yuan J, He G, Chen Y, Pan Q, Liu Y, et al: SOAPdenovo2: an empirically improved memory-efficient short-read de novo assembler. GigaScience 2012, 1(1):18.

3. Butler J, MacCallum I, Kleber M, Shlyakhter IA, Belmonte MK, Lander ES, Nusbaum C, Jaffe DB: ALLPATHS: de novo assembly of whole-genome shotgun microreads. Genome Res 2008, 18(5):810-820.

4. Parra G, Bradnam K, Ning Z, Keane T, Korf I: Assessing the gene space in draft genomes. Nucleic acids research 2009, 37(1):289-297.

5. Baker M: De novo genome assembly: what every biologist should know. Nature Methods 2012, 9:333-337.

6. Salzberg SL, Phillippy AM, Zimin A, Puiu D, Magoc T, Koren S, Treangen TJ, Schatz MC, Delcher AL, Roberts M, et al: GAGE: A critical evaluation of genome assemblies and assembly algorithms. Genome Res 2012, 22(3):557-567.

7. Earl D, Bradnam K, St John J, Darling A, Lin D, Fass J, Yu HO, Buffalo V, Zerbino DR, Diekhans $M$, et al: Assemblathon 1: a competitive assessment of de novo short read assembly methods. Genome Res 2011, 21(12):2224-2241.

8. Magoc T, Pabinger S, Canzar S, Liu X, Su Q, Puiu D, Tallon L, Salzberg SL: GAGE-B: An Evaluation of Genome Assemblers for Bacterial Organisms. Bioinformatics 2013.

9. Rawat A, Elasri MO, Gust KA, George G, Pham D, Scanlan LD, Vulpe C, Perkins EJ: CAPRG: sequence assembling pipeline for next generation sequencing of non-model organisms. PloS one 2012, 7(2):e30370.

10. Barriuso J, Valverde JR, Mellado RP: Estimation of bacterial diversity using next generation sequencing of 16S rDNA: a comparison of different workflows. BMC Bioinformatics 2011, 12:473.

11. Miller JR, Koren S, Sutton G: Assembly algorithms for next-generation sequencing data. Genomics 2010, 95(6):315-327.

12. Zhang $W$, Chen J, Yang $Y$, Tang $Y$, Shang J, Shen B: A practical comparison of de novo genome assembly software tools for next-generation sequencing technologies. PloS one 2011, 6(3):e17915.

13. Salzberg SL, Delcher AL, Kasif S, White O: Microbial gene identification using interpolated Markov models. Nucleic Acids Res 1998, 26(2):544-548.

14. Lukashin AV, Borodovsky M: GeneMark.hmm: new solutions for gene finding. Nucleic acids research 1998, 26(4):1107-1115.

15. Genome Annoucements. Genome Annoucements 2013, 1.

16. Begley CG, Ellis LM: Drug development: Raise standards for preclinical cancer research. Nature 2012, 483(7391):531-533.

17. Nekrutenko A, Taylor J: Next-generation sequencing data interpretation: enhancing reproducibility and accessibility. Nature reviews Genetics 2012 13(9):667-672

18. Leser SC-BaU: Next generation data integration for Life Sciences. IEEE 27th International Conference on Data Engineering 2011, 1366-1369.

19. Sandve GK, Nekrutenko A, Taylor J, Hovig E: Ten simple rules for reproducible computational research. PLoS Comput Biol 2013, 9(10): e1003285.

20. Deelman E, Gannon D, Shields M, Taylor I: Workflows and e-Science: An overview of workflow system features and capabilities. Future Generation Computer Systems 2009, 25(5):528-540.

21. Goecks J, Nekrutenko A, Taylor J: Galaxy: a comprehensive approach for supporting accessible, reproducible, and transparent computational research in the life sciences. Genome biology 2010, 11(8):R86.

22. Oinn T, Addis M, Ferris J, Marvin D, Senger M, Greenwood M, Carver T, Glover K, Pocock MR, Wipat A, et al: Taverna: a tool for the composition and enactment of bioinformatics workflows. Bioinformatics 2004 20(17):3045-3054.

23. Byelas HV MD, Swertz MA: Introducing Data Provenance and Error Handling For NGS Workflows Within the Molgenis Computational Framework. International Conference on Bioinformatics Models, Methods and Algorithms 2012

24. Kim HU, Kim SY, Jeong H, Kim TY, Kim JJ, Choy HE, Yi KY, Rhee JH, Lee SY: Integrative genome-scale metabolic analysis of Vibrio vulnificus for drug targeting and discovery. Mol Syst Biol 2011, 7:460.

25. Jones MK, Oliver JD: Vibrio vulnificus: disease and pathogenesis. Infect Immun 2009, 77(5):1723-1733.

26. Hall BG, Ehrlich GD, Hu FZ: Pan-genome analysis provides much higher strain typing resolution than multi-locus sequence typing. Microbiology 2010, 156(Pt 4):1060-1068. 
27. Kim YR, Lee SE, Kim CM, Kim SY, Shin EK, Shin DH, Chung SS, Choy HE, Progulske-Fox A, Hillman JD, et al: Characterization and pathogenic significance of Vibrio vulnificus antigens preferentially expressed in septicemic patients. Infection and immunity 2003, 71(10):5461-5471.

28. Aziz RK, Bartels D, Best AA, DeJongh M, Disz T, Edwards RA, Formsma K, Gerdes S, Glass EM, Kubal M, et al: The RAST Server: rapid annotations using subsystems technology. BMC genomics 2008, 9:75.

29. Li L, Stoeckert CJ, Roos DS: OrthoMCL: identification of ortholog groups for eukaryotic genomes. Genome Res 2003, 13(9):2178-2189.

30. Lander ES, Waterman MS: Genomic mapping by fingerprinting random clones: a mathematical analysis. Genomics 1988, 2(3):231-239.

31. Delcher AL, Salzberg SL, Phillippy AM: Using MUMmer to identify similar regions in large sequence sets. Curr Protoc Bioinformatics 2003, Chapter 10(Unit 10):13.

32. Nielsen $P$, Krogh A: Large-scale prokaryotic gene prediction and comparison to genome annotation. Bioinformatics 2005, 21(24):4322-4329.

33. Cain AA, Kosara R, Gibas CJ: GenoSets: visual analytic methods for comparative genomics. PloS one 2012, 7(10):e46401.

34. Ashburner M, Ball CA, Blake JA, Botstein D, Butler H, Cherry JM, Davis AP, Dolinski K, Dwight SS, Eppig JT, et al: Gene ontology: tool for the unification of biology. The Gene Ontology Consortium. Nature genetics 2000, 25(1):25-29.

35. Harris MA, Clark J, Ireland A, Lomax J, Ashburner M, Foulger R, Eilbeck K, Lewis S, Marshall B, Mungall C, et al: The Gene Ontology (GO) database and informatics resource. Nucleic acids research 2004, 32(Database): D258-261

36. Bauer S, Gagneur J, Robinson PN: GOing Bayesian: model-based gene set analysis of genome-scale data. Nucleic acids research 38(11):3523-3532.

37. Wright AC, Simpson LM, Oliver JD: Role of iron in the pathogenesis of Vibrio vulnificus infections. Infection and immunity 1981, 34(2):503-507.

38. Amaro C, Biosca EG, Fouz B, Toranzo AE, Garay E: Role of iron, capsule, and toxins in the pathogenicity of Vibrio vulnificus biotype 2 for mice. Infection and immunity 1994, 62(2):759-763.

39. Morrison SS, Williams T, Cain A, Froelich B, Taylor C, Baker-Austin C, VernerJeffreys D, Hartnell R, Oliver JD, Gibas CJ: Pyrosequencing-based comparative genome analysis of Vibrio vulnificus environmental isolates. PLoS One 2012, 7(5):e37553.

40. Field D, Garrity G, Gray T, Morrison N, Selengut J, Sterk P, Tatusova T, Thomson N, Allen MJ, Angiuoli SV, et al: The minimum information about a genome sequence (MIGS) specification. Nat Biotechnol 2008, 26(5):541-547.

41. Edgar R, Domrachev M, Lash AE: Gene Expression Omnibus: NCBI gene expression and hybridization array data repository. Nucleic acids research 2002, 30(1):207-210.

42. Haiminen N, Kuhn DN, Parida L, Rigoutsos I: Evaluation of methods for de novo genome assembly from high-throughput sequencing reads reveals dependencies that affect the quality of the results. PloS one 2011, 6(9): e24182.

43. Huang W, Li L, Myers JR, Marth GT: ART: a next-generation sequencing read simulator. Bioinformatics 2012, 28(4):593-594.

44. Andrews S: FastQC 2010

45. Conesa A, Gotz S, Garcia-Gomez JM, Terol J, Talon M, Robles M: Blast2GO: a universal tool for annotation, visualization and analysis in functional genomics research. Bioinformatics 2005, 21(18):3674-3676.

doi:10.1186/1471-2164-15-S8-S1

Cite this article as: Morrison et al: Impact of analytic provenance in genome analysis. BMC Genomics 2014 15(Suppl 8):S1.

\section{Submit your next manuscript to BioMed Central and take full advantage of:}

- Convenient online submission

- Thorough peer review

- No space constraints or color figure charges

- Immediate publication on acceptance

- Inclusion in PubMed, CAS, Scopus and Google Scholar

- Research which is freely available for redistribution 